\title{
Computationally Efficient DOA and Polarization Estimation of Coherent Sources with Linear Electromagnetic Vector-Sensor Array
}

\author{
Zhaoting Liu, ${ }^{1}$ Jing He, ${ }^{2}$ and Zhong Liu ${ }^{1}$ \\ ${ }^{1}$ Department of Electronic Engineering, Nanjing University of Science and Technology, Nanjing, Jiangsu 210094, China \\ ${ }^{2}$ Department of Electrical and Computer Engineering, Concordia University, Montreal, QC, Canada H3G 2W1
}

Correspondence should be addressed to Zhaoting Liu, liuzhaoting@163.com

Received 3 September 2010; Revised 10 December 2010; Accepted 16 January 2011

Academic Editor: Ana Pérez-Neira

Copyright (C) 2011 Zhaoting Liu et al. This is an open access article distributed under the Creative Commons Attribution License, which permits unrestricted use, distribution, and reproduction in any medium, provided the original work is properly cited.

\begin{abstract}
This paper studies the problem of direction finding and polarization estimation of coherent sources using a uniform linear electromagnetic vector-sensor (EmVS) array. A novel preprocessing algorithm based on EmVS subarray averaging (EVSA) is firstly proposed to decorrelate sources' coherency. Then, the proposed EVSA algorithm is combined with the propagator method (PM) to estimate the EmVS steering vector, and thus estimate the direction-of-arrival (DOA) and the polarization parameters by a vector cross-product operation. Compared with the existing estimate methods, the proposed EVSA-PM enables decorrelation of more coherent signals, joint estimation of the DOA and polarization of coherent sources with a lower computational complexity, and requires no limitation of the intervector sensor spacing within a half-wavelength to guarantee unique and unambiguous angle estimates. Also, the EVSA-PM can estimate these parameters by parameter-space searching techniques. Monte-Carlo simulations are presented to verify the efficacy of the proposed algorithm.
\end{abstract}

\section{Introduction}

A typical electromagnetic vector-sensor (EmVS) consists of six component sensors configured by two orthogonal triads of dipole and loop antennas with the same phase center. Therefore, an EmVS can simultaneously measure the three components of the electric field and the three components of the magnetic field. Since its introduction into signal processing community $[1,2]$, a significant number of research has been done on EmVS array processing [3-19]. For application considerations, different types of EmVS containing part of the six sensors are devised and manufactured $[3,20,21]$.

In the study of direction finding applications, conventional eigenstructure-based source localization techniques have been extended to the case of the EmVS array. ESPRIT/ MUSIC algorithms using EmVS arrays obtain thorough investigations $[10-12,16-19]$. The signal subspace and noise subspace are usually constructed by decomposing the column space of the data correlation matrix with the eigen-decomposition (or singular value decomposition) techniques $[22,23]$. Because the decomposing process is computationally intensive and time consuming, the eigenstructure-based techniques may be unsuitable for many practical situations, especially when the number of vector sensors is large and/or the directions of impinging sources should be tracked in an online manner.

Furthermore, the eigenstructure-based direction finding techniques using the EmVS arrays usually assume incoherent signals, that is, that the signal covariance matrix has full rank. This assumption is often violated in scenarios where multipath exists. Coherent signals could reduce the rank of signal covariance matrix below the number of incident signals, and hence, degrade critically the algorithmic performance. 
To deal with the coherent signals using the EmVS array, a polarization smoothing algorithm (PSA) has been proposed to restore the rank of signal subspace [19]. The PSA does not reduce the effective array aperture length and has no limit to array geometries. However, the PSA-based method has nonnegligible drawbacks. (1) It assumes the intervector sensor spacing within a half-wavelength to guarantee unique and unambiguous angle estimates; (2) it is not able to estimate the polarization of impinging electromagnetic waves; (3) the EmVS type limits the maximum number of resolvable coherent signals.

In this paper, we employ a uniform linear EmVS array to perform parameter estimation of coherent sources. Firstly, to decorrelate the coherent sources, an EmVS subarray averaging-based pre-processing (EVSA) algorithm is developed. Then the EVSA algorithm is coupled with the propagator method (PM) $[24,25]$ to estimate parameters of the coherent sources without eigen-decomposition or singular value decomposition unlike the ESPRIT/MUSICbased methods. By using the vector cross-product of the electric field vector estimate and the magnetic field vector estimate, the proposed EVSA-PM can estimate both the DOA and polarization parameters, hence, can overcome the drawbacks of the PSA-based algorithms to some extent. The vector cross-product estimator is valid to a six-component EmVS array. For the array comprising any types of EmVSs, the EVSA-PM with parameter-space searching techniques is developed to estimate the parameters. The EVSA-PM can be regarded as an extension of the subspace-based method without eigendecomposition (SUMWE) [26] to the case of the EmVS arrays. The SUMWE is also a PM-based method, which estimates the DOA of coherent sources using unpolarized scalar sensors by an iterative angle searching. However, the proposed methods make use of more available electromagnetic information, and hence, should outperform the SUMWE algorithm in accuracy and resolution of DOA estimation.

The rest of this paper is organized as follows. Section 2 formulates the mathematical data model of EmVS array. Section 3 develops the proposed EmVS-PM. Section 4 presents the simulation results to verify the efficacy of the EmVS-PM. Section 5 concludes the paper.

\section{Mathematical Data Model}

Assume that $K$ narrowband completely polarized coherent signals impinge upon a uniform linear EmVS array with $M$ vector sensors $(M>2 K)$, and the array is neither mutual coupling nor cross-polarization effects. The $K$ is known in advance and the $k$ th incident source is parameterized $\left\{\theta_{k}, \varphi_{k}, \gamma_{k}, \eta_{k}\right\}$, where $0 \leq \theta_{k} \leq \pi / 2$ denotes the $k$ th source's elevation angle measured from the vertical $z$-axis, $0 \leq \varphi_{k} \leq$ $2 \pi$ represents the $k$ th source's azimuth angle, $0 \leq \gamma_{k} \leq \pi / 2$ refers to the $k$ th source's auxiliary polarization angle, and $-\pi \leq \eta_{k} \leq \pi$ symbolizes the $k$ th source's polarization phase difference. For a six-component EmVS, the steering vector of the $k$ th unit-power electromagnetic source signal produces the following $6 \times 1$ vector:

$$
\begin{aligned}
& \mathbf{c}\left(\theta_{k}, \varphi_{k}, \gamma_{k}, \eta_{k}\right) \\
& \stackrel{\text { def }}{=}\left[\begin{array}{c}
c_{1, k} \\
c_{2, k} \\
c_{3, k} \\
c_{4, k} \\
c_{5, k} \\
c_{6, k}
\end{array}\right] \stackrel{\text { def }}{=}\left[\begin{array}{c}
e_{x, k} \\
e_{y, k} \\
e_{z, k} \\
h_{x, k} \\
h_{y, k} \\
h_{z, k}
\end{array}\right] \\
& =\underbrace{\left[\begin{array}{cc}
\cos \varphi_{k} \cos \theta_{k} & -\sin \varphi_{k} \\
\sin \varphi_{k} \cos \theta_{k} & \cos \varphi_{k} \\
-\sin \theta_{k} & 0 \\
-\sin \varphi_{k} & -\cos \varphi_{k} \cos \theta_{k} \\
\cos \varphi_{k} & -\sin \varphi_{k} \cos \theta_{k} \\
0
\end{array}\right]}_{\underset{\operatorname{def}}{=} \Theta\left(\theta_{k}, \phi_{k}\right)} \underbrace{\stackrel{\text { def }}{=} \mathbf{g}\left(\gamma_{k}, \eta_{k}\right)}_{\left.\begin{array}{c}
\sin \gamma_{k} e^{j \eta_{k}} \\
\cos \gamma_{k}
\end{array}\right]},
\end{aligned}
$$

where $\mathbf{e}_{k} \stackrel{\text { def }}{=}\left[e_{x, k}, e_{y, k}, e_{z, k}\right]^{T}$ and $\mathbf{h}_{k} \stackrel{\text { def }}{=}\left[h_{x, k}, h_{y, k}, h_{z, k}\right]^{T}$ denote the electric field vector and the magnetic field vector, respectively.

The intersensor spatial phase factor for the $k$ th incident signal and the $m$ th vector sensor is $q_{m}\left(\theta_{k}, \varphi_{k}\right) \stackrel{\text { def }}{=}$ $e^{j 2 \pi\left(x_{m} u_{k}+y_{m} v_{k}\right) / \lambda}$, where $u_{k} \stackrel{\text { def }}{=} \sin \theta_{k} \cos \varphi_{k}$ and $v_{k} \stackrel{\text { def }}{=}$ $\sin \theta_{k} \sin \varphi_{k}$ signify the direction cosines along the $x$-axis and $y$-axis, respectively. $\left(x_{m}, y_{m}\right)$ is the location of the $m$ th vector sensor, $\lambda$ equals the signals' wavelength. Denoting the spacing between adjacent vector sensors as $\left(\Delta_{x}, \Delta_{y}\right)$, we have $x_{m}=x_{1}+(m-1) \Delta_{x}, y_{m}=y_{1}+(m-1) \Delta_{y}$. The $6 \times 1$ measurement vector corresponding to the $m$ th vector sensor can be expressed as

$$
\begin{aligned}
\mathbf{x}_{m}(t) & \stackrel{\text { def }}{=}\left[x_{m, 1}(t), \ldots, x_{m, 6}(t)\right]^{T} \\
& =\sum_{k=1}^{K} q_{m}\left(\theta_{k}, \varphi_{k}\right) \mathbf{c}\left(\theta_{k}, \varphi_{k}, \gamma_{k}, \eta_{k}\right) s_{k}(t)+\mathbf{w}_{m}(t),
\end{aligned}
$$

where $\mathbf{w}_{m}(t)=\left[w_{m, 1}(t), \ldots, w_{m, 6}(t)\right]^{T}$ is the additive zeromean complex noise and independent to all signals. $x_{m, n}(t)$ and $w_{m, n}(t)$ refer to the measurement and the noise corresponding to the $m$ th vector sensor's $n$th component, respectively; $s_{k}(t)$ represents the $k$ th source's complex envelope. Without loss of generality, we consider the signals $\left\{s_{k}(t)\right\}$ are all coherent so that they are all some complex multiples of a common signal $s_{1}(t)$. Then, under the flat-fading multipath propagation, they can be expressed as $s_{k}(t)=\beta_{k} s_{1}(t)[26,27]$, where $\beta_{k}$ is the multipath coefficient that represents the complex attenuation of the $k$ th signal with respect to the first one $\left(\beta_{1}=1\right.$ and $\left.\beta_{k} \neq 0\right)$. 
For the entire vector-sensor array, the array manifold, $\mathbf{a}\left(\theta_{k}, \varphi_{k}, \gamma_{k}, \eta_{k}\right) \in C^{6 M \times 1}$, is given by

$$
\mathbf{a}\left(\theta_{k}, \varphi_{k}, \gamma_{k}, \eta_{k}\right) \stackrel{\text { def }}{=} \mathbf{q}\left(\theta_{k}, \varphi_{k}\right) \otimes \mathbf{c}\left(\theta_{k}, \varphi_{k}, \gamma_{k}, \eta_{k}\right),
$$

where $\otimes$ symbolizes the Kronecker product operator, $\mathbf{q}\left(\theta_{k}, \varphi_{k}\right) \stackrel{\text { def }}{=}\left[q_{1}\left(\theta_{k}, \varphi_{k}\right), \ldots, q_{M}\left(\theta_{k}, \varphi_{k}\right)\right]^{T}$. With a total of $K$ signals, the entire $6 M \times 1$ output vector measured by the EmVS array at time $t$ has the complex envelope represented as

$$
\begin{aligned}
\mathbf{z}(t) & =\left[\mathbf{x}_{1}^{T}(t), \ldots, \mathbf{x}_{M}^{T}(t)\right]^{T} \\
& =\sum_{k=1}^{K} \mathbf{a}\left(\theta_{k}, \varphi_{k}, \gamma_{k}, \eta_{k}\right) s_{k}(t)+\mathbf{n}(t) \\
& =\mathbf{A s}(t)+\mathbf{n}(t),
\end{aligned}
$$

where $\mathbf{A} \in C^{6 M \times K}, \mathbf{s}(t) \in C^{K \times 1}, \mathbf{n}(t) \in C^{6 M \times 1}$, and $\mathbf{A}=$ $\left[\mathbf{a}\left(\theta_{1}, \varphi_{1}, \gamma_{1}, \eta_{1}\right), \ldots, \mathbf{a}\left(\theta_{K}, \varphi_{K}, \gamma_{K}, \eta_{K}\right)\right] ; \mathbf{s}(t)=\left[s_{1}(t), \ldots\right.$, $\left.s_{K}(t)\right]^{T}, \mathbf{n}(t)=\left[\mathbf{w}_{1}^{T}(t), \ldots, \mathbf{w}_{M}^{T}(t)\right]^{T}$.

\section{Algorithm Development}

This section is devoted to the algorithm development. Section 3.1 develops the EVSA algorithm, Section 3.2 describes EVSA-PM algorithm for estimating both DOA and polarization parameters from the available EmVS steering vector estimates and Section 3.3 is for parameters estimation by parameter-space searching techniques.

3.1. EVSA Algorithm. Let us consider the subarray averaging scheme with a linear EmVS array, which is divided into $L$ overlapping subarrays with $K$ vector sensors and the $l$ th subarray comprises the $l$ th to $(l+K-1)$ th vector sensor, where $L=M-K+1$. We use the first vector sensor as a reference $\left(x_{1}=0, y_{1}=0\right)$, and then the corresponding $6 K \times 1$ signal vector is given as

$$
\mathbf{z}_{l}(t) \stackrel{\text { def }}{=}\left[\mathbf{x}_{l}^{T}(t), \ldots, \mathbf{x}_{l+K-1}^{T}(t)\right]^{T}=\mathbf{A}_{0} \mathbf{D}^{l-1} \mathbf{s}(t)+\mathbf{n}_{l}(t),
$$

where $\mathbf{D} \in C^{K \times K}$, and $\mathbf{D} \stackrel{\text { def }}{=} \operatorname{diag}\left(e^{j 2 \pi\left(\Delta_{x} u_{1}+\Delta_{y} v_{1}\right) / \lambda}, \ldots\right.$, $\left.e^{j 2 \pi\left(\Delta_{x} u_{K}+\Delta_{y} v_{K}\right) / \lambda}\right) ; \mathbf{A}_{0} \in C^{6 K \times K}$ contains the first $6 K$ rows of $\mathbf{A}$; $\mathbf{n}_{l}(t) \stackrel{\text { def }}{=}\left[\mathbf{w}_{l}^{T}(t), \ldots, \mathbf{w}_{l+K-1}^{T}(t)\right]^{T}$. We can calculate the crosscorrelation vector $\boldsymbol{\varphi}_{l, n} \in C^{6 K \times 1}$ between $\mathbf{z}_{l}(t)$ and $x_{M, n}(t)$

$$
\begin{aligned}
\boldsymbol{\varphi}_{l, n} \stackrel{\text { def }}{=} E\left\{\mathbf{z}_{l}(t) x_{M, n}^{*}(t)\right\} \\
\\
=\mathbf{A}_{0} \mathbf{D}^{l-1} E\left\{\mathbf{s}(t) \mathbf{s}^{H}(t)\right\} \mathbf{a}_{M, n}^{*}+E\left\{\mathbf{n}_{l}(t) w_{M, n}^{*}\right\} \\
=\rho_{M, n} r_{s} \mathbf{A}_{0} \mathbf{D}^{l-1} \boldsymbol{\beta}, \quad l=1, \ldots, L-1 ; n=1, \ldots, 6,
\end{aligned}
$$

where $E\{\cdot\}$ denotes the expectation, $r_{s} \stackrel{\text { def }}{=} E\left\{s_{1}(t) s_{1}^{*}(t)\right\}$, $\rho_{l, n} \stackrel{\text { def }}{=} \boldsymbol{\beta}^{H} \mathbf{a}_{l, n}^{*}, \mathbf{a}_{l, n} \stackrel{\text { def }}{=}\left[q_{l}\left(\theta_{1}, \varphi_{1}\right) c_{n, 1}, \ldots, q_{l}\left(\theta_{K}, \varphi_{K}\right) c_{n, K}\right]^{T}$, $\boldsymbol{\beta} \stackrel{\text { def }}{=}\left[\beta_{1}, \ldots, \beta_{K}\right]^{T}$. Similarly, the cross-correlation vector $\widetilde{\boldsymbol{\varphi}}_{l, n} \in C^{6 K \times 1}$ between $\mathbf{z}_{l}(t)$ and $x_{1, n}(t)$ is as follows

$$
\begin{aligned}
\tilde{\boldsymbol{\varphi}}_{l, n} & \stackrel{\text { def }}{=} E\left\{\mathbf{z}_{l}(t) x_{1, n}^{*}(t)\right\} \\
& =\rho_{1, n} r_{s} \mathbf{A}_{0} \mathbf{D}^{l-1} \boldsymbol{\beta}, \quad l=2, \ldots, L ; n=1, \ldots, 6 .
\end{aligned}
$$

Let us rewrite the vector $\varphi_{l, n}$ as a $6 \times K$ matrix

$$
\begin{aligned}
\boldsymbol{\Phi}_{l, n} & \stackrel{\text { def }}{=}\left[\mathbf{J}_{1} \boldsymbol{\varphi}_{l, n}, \ldots, \mathbf{J}_{K} \boldsymbol{\varphi}_{l, n}\right] \\
& =\rho_{M, n} r_{s}\left[\widetilde{\mathbf{A}}_{l} \mathbf{D}^{l-1} \boldsymbol{\beta}, \ldots, \widetilde{\mathbf{A}}_{K} \mathbf{D}^{l-1} \boldsymbol{\beta}\right] \\
& =\rho_{M, n} r_{s} \widetilde{\mathbf{A}}_{l}\left[\boldsymbol{\beta}, \ldots, \mathbf{D}^{K-1} \boldsymbol{\beta}\right] \\
& =\rho_{M, n} r_{s} \widetilde{\mathbf{A}}_{l} \mathbf{B} \mathbf{Q}^{T},
\end{aligned}
$$

where $\mathbf{J}_{k} \stackrel{\text { def }}{=}\left[\mathbf{0}_{6,6(k-1)}, \mathbf{I}_{6}, \mathbf{0}_{6,6(K-k)}\right] ; \mathbf{B} \stackrel{\text { def }}{=} \operatorname{diag}\left(\beta_{1}, \ldots, \beta_{K}\right)$; $\tilde{\mathbf{A}}_{l}$ is the $6 \times K$ matrix with the column $\mathbf{c}_{k} q_{l}\left(\theta_{k}, \varphi_{k}\right)$, $k=1, \ldots, 6$; $\mathbf{Q}$ is the $K \times K$ matrix with the column $\left[q_{1}\left(\theta_{k}, \varphi_{k}\right), \ldots, q_{K}\left(\theta_{k}, \varphi_{k}\right)\right]^{T}$. Similarly, the vector $\widetilde{\boldsymbol{\varphi}}_{l, n}$ can be rewritten as

$$
\widetilde{\boldsymbol{\Phi}}_{l, n} \stackrel{\text { def }}{=}\left[\mathbf{J}_{1} \widetilde{\boldsymbol{\varphi}}_{l, n}, \ldots, \mathbf{J}_{K} \widetilde{\boldsymbol{\varphi}}_{l, n}\right]=\rho_{1, n} r_{s} \tilde{\mathbf{A}}_{l} \mathbf{B} \mathbf{Q}^{T} .
$$

Therefore, concatenating $\boldsymbol{\Phi}_{l, n}$ for $l=1, \ldots, L-1$ and $\widetilde{\boldsymbol{\Phi}}_{l, n}$ for $l=2, \ldots, L$, respectively, we can get two correlation matrices

$$
\begin{gathered}
\mathbf{R}_{n} \stackrel{\text { def }}{=}\left[\boldsymbol{\Phi}_{1, n}^{T}, \boldsymbol{\Phi}_{2, n}^{T}, \ldots, \boldsymbol{\Phi}_{(L-1), n}^{T}\right]^{T}=\rho_{M, n} r_{s} \widetilde{\mathbf{A}} \mathbf{B} \mathbf{Q}^{T}, \\
\widetilde{\mathbf{R}}_{n} \stackrel{\text { def }}{=}\left[\widetilde{\boldsymbol{\Phi}}_{2, n}^{T}, \widetilde{\boldsymbol{\Phi}}_{3, n}^{T}, \ldots, \widetilde{\boldsymbol{\Phi}}_{L, n}^{T}\right]^{T}=\rho_{1, n} r_{s} \widetilde{\mathbf{A}} \mathbf{B D Q} \mathbf{Q}^{T},
\end{gathered}
$$

where $\mathbf{R}_{n} \in C^{6(L-1) \times K}, \widetilde{\mathbf{R}}_{n} \in C^{6(L-1) \times K}$, and $\widetilde{\mathbf{A}} \stackrel{\text { def }}{=}\left[\widetilde{\mathbf{A}}_{1}^{T}, \ldots\right.$, $\left.\tilde{\mathbf{A}}_{L-1}^{T}\right]^{T}$ includes the first $6(L-1)$ rows of $\mathbf{A}$. With $(10)$, the EmVS subarray averaging (EVSA) matrix can be formulated as

$$
\mathbf{R} \stackrel{\text { def }}{=}\left[\mathbf{R}_{1}, \ldots, \mathbf{R}_{6}, \widetilde{\mathbf{R}}_{1}, \ldots, \widetilde{\mathbf{R}}_{6}\right]=\tilde{\mathbf{A}} \boldsymbol{\Omega},
$$

where $\boldsymbol{\Omega} \stackrel{\text { def }}{=} r_{s} \mathbf{B}\left[\rho_{M, 1} \mathbf{Q}^{T}, \ldots, \rho_{M, 6} \mathbf{Q}^{T}, \rho_{1,1} \mathbf{D} \mathbf{Q}^{T}, \ldots, \rho_{1,6} \mathbf{D Q}^{T}\right]$. Note that $\mathbf{B}$ and $\mathbf{D}$ are diagonal matrices with nonzero diagonal elements, and $\mathbf{Q}$ is full rank when all sources impinge with the distinct incident directions. Then the $\mathbf{R}_{n}$ and $\widetilde{\mathbf{R}}_{n}$ are of rank $K$, and hence, $\mathbf{R}$ is of rank $K$ and can be used to estimate the DOA and the polarization parameters of the coherent sources.

In realistic cases where only a finite number of snapshots are available, the cross-correlation vector $\boldsymbol{\varphi}_{l, n}$ and $\widetilde{\varphi}_{l, n}$ can be estimated as $\hat{\boldsymbol{\varphi}}_{l, n}=\sum_{t=1}^{S} \mathbf{z}_{l}(t) x_{M, n}^{*}(t) / S$ and $\hat{\widetilde{\varphi}}_{l, n}=$ $\sum_{t=1}^{S} \mathbf{z}_{l}(t) x_{1, n}^{*}(t) / S$, where $S$ denotes the number of snapshots. With $\hat{\boldsymbol{\varphi}}_{l, n}$ and $\hat{\widetilde{\varphi}}_{l, n}$, the matrix $\mathbf{R}$ is accordingly obtained using (8)-(11).

Note that the proposed EVSA algorithm can also be used to the case of partly coherent or incoherent signals. To see this, we assume that the first $K_{1}\left(1 \leq K_{1} \leq K\right)$ incident 
signals are coherent and the others are uncorrelated with these signals and with each other. Then after some algebraic manipulations, we can obtain

$$
\begin{gathered}
\mathbf{R}_{n}=\tilde{\rho}_{M, n} r_{s_{1}} \tilde{\mathbf{A}} \widetilde{\mathbf{B}} \mathbf{Q}^{T}+\tilde{\mathbf{A}} \widetilde{\mathbf{R}} \mathbf{A}_{M, n}^{H} \mathbf{Q}^{T}, \\
\widetilde{\mathbf{R}}_{n}=\tilde{\rho}_{1, n} r_{s_{1}} \tilde{\mathbf{A}} \widetilde{\mathbf{B}} \mathbf{D} \mathbf{Q}^{T}+\widetilde{\mathbf{A}} \mathbf{D} \widetilde{\mathbf{R}} \mathbf{A}_{1, n}^{H} \mathbf{Q}^{T},
\end{gathered}
$$

where $\tilde{\rho}_{l, n} \stackrel{\text { def }}{=} \widetilde{\boldsymbol{\beta}}^{H} \mathbf{a}_{l, n}^{*}, \widetilde{\boldsymbol{\beta}} \stackrel{\text { def }}{=}\left[\beta_{1}, \ldots, \beta_{K_{1}}, 0, \ldots, 0\right]^{T}, \widetilde{\mathbf{B}} \stackrel{\text { def }}{=}$ $\operatorname{diag}\left(\beta_{1}, \ldots, \beta_{K_{1}}, 0, \ldots, 0\right), r_{s_{k}} \stackrel{\text { def }}{=} E\left\{s_{k}(t) s_{k}^{*}(t)\right\}, \widetilde{\mathbf{R}} \stackrel{\text { def }}{=} \operatorname{diag}(0$, $\left.\ldots, r_{s_{K_{1}+1}}, \ldots, r_{s_{K}}\right), \quad \mathbf{A}_{l, \mathrm{n}} \stackrel{\text { def }}{=} \operatorname{diag}\left(q_{l}\left(\theta_{1}, \varphi_{1}\right) c_{n, 1}, \ldots, q_{l}\left(\theta_{K}\right.\right.$, $\left.\left.\varphi_{K}\right) c_{n, K}\right)$. It is easy to find that the rank of $\mathbf{R}_{n}$ and $\widetilde{\mathbf{R}}_{n}$ still equals $K$ when all sources impinge with the distinct incident directions.

Remarks. (1) The proposed EVSA algorithm is still effective in the case of partly coherent or incoherent sources in which there exist two incoherent sources with the same incident directions but with the distinct polarizations. As shown in the appendix, the matrix $\mathbf{R}$ defined in (11) has full rank. However, neither the PSA [19] nor the SUMWE [26] algorithm can be so.

(2) The EVSA algorithm needs low computations. As seen from (6) and (7), the EVSA only needs compute the cross-correlations, which require $72(L-1)$ cross-correlation operations. However, most of EmVS direction finding algorithms require to compute the correlations of all array data with $(6 M)^{2}$ correlation operations.

(3) The EVSA-based method may estimate both DOA and polarization parameters, while the PSA-based one can only estimate the DOA parameters because of the polarization smoothing.

(4) From (11), the EVSA algorithm can decorrelate more coherent sources than the PSA can do. The EVSA algorithm can decorrelate up-to $L-2$ coherent sources regardless of EmVS's types, while the PSA can only decorrelate 6 coherent sources for six-component EmVS array, 4 for quadrature polarized array [19] and 2 for dual polarized array [19]. By coupling the forward/backward (FB) averaging technique [27], the maximum number of the coherent signals decorrelated by the PSA is doubled, however, it is only valid for the case of the symmetric array, for instance, uniform linear array, to which the proposed method is limited.

3.2. EVSA-PM Algorithm for Estimating Parameters from the EmVS Steering Vector. The EVSA-PM algorithm performs the estimation of the coherent sources' DOA and polarization parameters by using the vector cross-product operation of the estimated electric field vector and magnetic field vector. For this purpose, we define an exchange matrix

$$
\begin{aligned}
\mathbf{E}= & {\left[\mathbf{e}_{1}, \mathbf{e}_{7}, \ldots, \mathbf{e}_{6(L-2)+1}, \mathbf{e}_{2}, \mathbf{e}_{8}, \ldots, \mathbf{e}_{6(L-2)+2}, \ldots,\right.} \\
& \left.\mathbf{e}_{6}, \mathbf{e}_{12}, \ldots, \mathbf{e}_{6(L-1)}\right],
\end{aligned}
$$

where $\mathbf{e}_{i}$ is the 6(L-1) dimensional unit vector whose $i$ th element is 1 and other elements are zero. In addition, we define

$$
\mathbf{R}_{\mathrm{e}} \stackrel{\text { def }}{=} \mathbf{E}^{T} \mathbf{R}=\mathbf{A}_{\mathrm{e}} \boldsymbol{\Omega},
$$

$$
\mathbf{A}_{\mathrm{e}} \stackrel{\text { def }}{=} \mathbf{E}^{T} \tilde{\mathbf{A}}=\left[\mathbf{A}_{\mathrm{e}, 1}^{T}, \ldots, \mathbf{A}_{\mathrm{e}, 6}^{T}\right]^{T},
$$

where $\mathbf{A}_{\mathrm{e}} \in C^{6(L-1) \times K}, \mathbf{A}_{\mathrm{e}, n} \in C^{(L-1) \times K}(n=1, \ldots, 6)$ is a submatrix whose $k$ th column is given as $\mathbf{q}_{\mathrm{e}}\left(\theta_{k}, \varphi_{k}\right) c_{n, k}$ with $\mathbf{q}_{\mathrm{e}}\left(\theta_{k}, \varphi_{k}\right) \stackrel{\text { def }}{=}\left[q_{1}\left(\theta_{k}, \varphi_{k}\right), \ldots, q_{L-1}\left(\theta_{k}, \varphi_{k}\right)\right]^{T}$. These submatrices are related with each other by

$$
\mathbf{A}_{e, n}=\mathbf{A}_{e, 1} \Lambda_{n}
$$

where $\boldsymbol{\Lambda}_{n} \in C^{K \times K}$ and $\boldsymbol{\Lambda}_{n} \stackrel{\text { def }}{=} \operatorname{diag}\left(d_{n, 1}, \ldots, d_{n, K}\right)$ with $d_{n, k} \stackrel{\text { def }}{=}$ $c_{n, k} / c_{1, k}$ denoting the $k$ th source's invariant factor between the first and the $n$th EmVS component.

We can divide $\mathbf{A}_{e, n}$ into

$$
\mathbf{A}_{e, n}=\left[\begin{array}{l}
\mathbf{A}_{e, n}^{(1)} \\
\mathbf{A}_{e, n}^{(2)}
\end{array}\right], \quad n=1, \ldots, 6,
$$

where $\mathbf{A}_{e, n}^{(1)} \in C^{K \times K}$ and $\mathbf{A}_{e, n}^{(2)} \in C^{(L-1-K) \times K}$. Therefore, $\mathbf{A}_{\mathrm{e}, n}$ can be rewritten as

$$
\mathbf{A}_{\mathrm{e}}=\left[\begin{array}{c}
\mathbf{A}_{\mathrm{e}, 1}^{(1)} \\
\mathbf{U}
\end{array}\right],
$$

where $\mathbf{U} \stackrel{\text { def }}{=}\left[\left(\mathbf{A}_{\mathrm{e}, 1}^{(2)}\right)^{T},\left(\mathbf{A}_{\mathrm{e}, 2}^{(1)}\right)^{T},\left(\mathbf{A}_{\mathrm{e}, 2}^{(2)}\right)^{T}, \ldots,\left(\mathbf{A}_{\mathrm{e}, 6}^{(1)}\right)^{T},\left(\mathbf{A}_{\mathrm{e}, 6}^{(2)}\right)^{T}\right]^{T}$. Obviously, $\mathbf{A}_{\mathrm{e}, n}^{(1)}$ is a matrix with full rank. The $K \times(6 L-6-$ $K)$ propagator matrix $\mathbf{P}$ can be defined as a unique linear operator which relates the matrices $\mathbf{A}_{\mathrm{e}, 1}^{(1)}$ and $\mathbf{U}$ through the equation

$$
\mathbf{P}^{H} \mathbf{A}_{\mathrm{e}, 1}^{(1)}=\mathbf{U} .
$$

We partition $\mathbf{P}^{H}$ into $\mathbf{P}^{H}=\left[\mathbf{P}_{1}^{T}, \mathbf{P}_{2}^{T}, \ldots, \mathbf{P}_{11}^{T}\right]^{T}$, where $\mathbf{P}_{1}$ to $\mathbf{P}_{11}$ have the dimensions identical to $\mathbf{A}_{e, 1}^{(2)}, \mathbf{A}_{e, 2}^{(1)}, \mathbf{A}_{e, 2}^{(2)}, \mathbf{A}_{e, 3}^{(1)}, \mathbf{A}_{e, 3}^{(2)}$, $\mathbf{A}_{e, 4}^{(1)}, \mathbf{A}_{e, 4}^{(2)}, \mathbf{A}_{e, 5}^{(1)}, \mathbf{A}_{e, 5}^{(2)}, \mathbf{A}_{e, 6}^{(1)}$, and $\mathbf{A}_{e, 6}^{(2)}$, respectively. Thus, we have

$$
\mathbf{P}_{1} \mathbf{A}_{e, 1}^{(1)}=\mathbf{A}_{e, 1}^{(2)},
$$

$$
\mathbf{P}_{2 n-1} \mathbf{A}_{e, 1}^{(1)}=\mathbf{A}_{e, 1}^{(2)} \boldsymbol{\Lambda}_{n}, \quad n=2, \ldots, 6 .
$$

Equations (20) and (21) together yield

$$
\mathbf{P}_{1}^{\dagger} \mathbf{P}_{2 n-1}=\mathbf{A}_{e, 1}^{(1)} \boldsymbol{\Lambda}_{n}\left(\mathbf{A}_{e, 1}^{(1)}\right)^{-1}, \quad n=2, \ldots, 6,
$$

where $\uparrow$ denotes the Pseudo inverse.

Equation (22) suggests that the matrices $\mathbf{P}_{1}^{\dagger} \mathbf{P}_{2 n-1}(n=$ $2, \ldots, 6)$ have the same set of eigenvectors and the corresponding eigenvalues lead to the invariant factors of the same sources. Hence, we can obtain the eigenvalue pairs by 


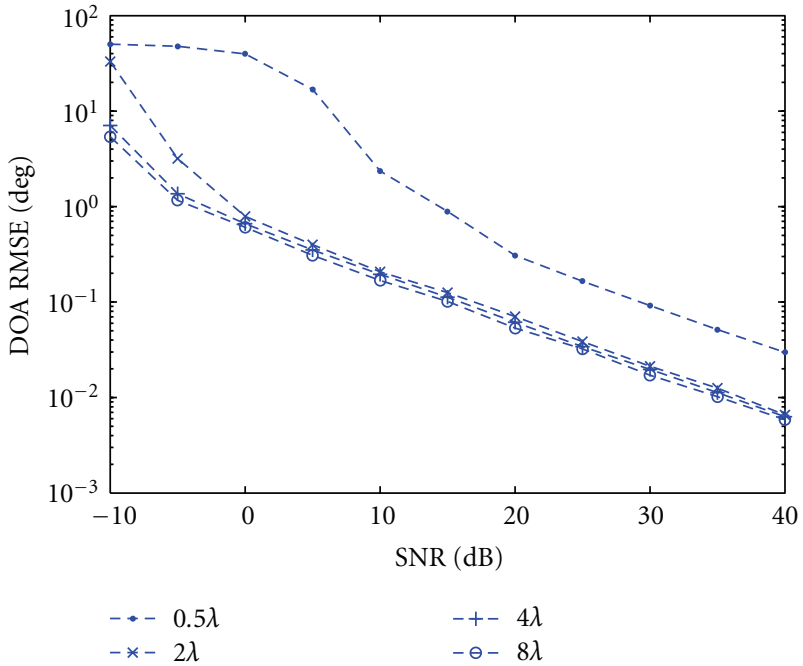

(a)

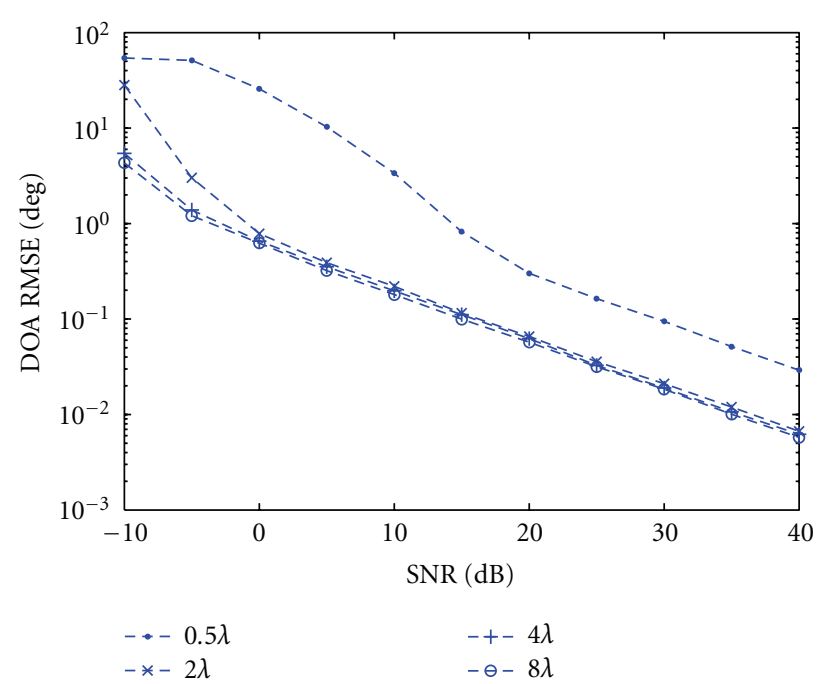

(b)

FIgURe 1: DOA estimates RMSE of the proposed EVSA-PM against SNRs. (a) Source 1, (b) source 2.

matching the eigenvectors of the different matrices $\mathbf{P}_{1}^{\dagger} \mathbf{P}_{2 n-1}$ $(n=2, \ldots, 6)$ [11]. With the estimated $\widehat{\mathbf{c}}\left(\theta_{k}, \varphi_{k}, \gamma_{k}, \eta_{k}\right)=$ $\left[1, \hat{d}_{2, k}, \ldots, \hat{d}_{6, k}\right]^{T}$, the Poynting vector estimates can be obtained by the vector cross-product operation and then the DOA and polarization parameters are estimated from the normalized Poynting vectors [11]. For a dipole triad array or loop triad array, the estimates of the electric field vector $\mathbf{e}_{k}$ or the magnetic field vector $\mathbf{h}_{k}$ can be done in the same way. In this case, the DOA and polarization parameter estimates can be obtained using the amplitude-normalized estimates of the electric or magnetic field steering vector [3].

In order to calculate the propagator matrix $\mathbf{P}$, we divide the matrix $\mathbf{R}_{\mathrm{e}}$ into $\mathbf{R}_{\mathrm{e}}=\left[\mathbf{R}_{\mathrm{e} 1}^{T}, \mathbf{R}_{\mathrm{e} 2}^{T}\right]^{T}$, where $\mathbf{R}_{\mathrm{e} 1}$ and $\mathbf{R}_{\mathrm{e} 2}$ consist of the first $K$ rows and the last $6 L-6-K$ rows of $\mathbf{R}_{\mathrm{e}}$. In the noise-free case, we have $\mathbf{P}^{H} \mathbf{R}_{\mathrm{e} 1}=\mathbf{R}_{\mathrm{e} 2}$. In the noise case, a least squares solution can be used to estimate $\mathbf{P}$

$$
\widehat{\mathbf{P}}=\left(\mathbf{R}_{\mathrm{e} 1} \mathbf{R}_{\mathrm{e} 1}^{H}\right)^{-1} \mathbf{R}_{\mathrm{e} 1} \mathbf{R}_{\mathrm{e} 2}^{H} .
$$

3.3. EVSA-PM Algorithm for Estimating Parameters by Angle Searching. The EVSA-PM is also applied to the uniform linear array comprising any types of identical EmVSs. In the case, the estimates of DOA and polarization parameters cannot be extracted from the estimates of the steering vectors. However, they are obtainable by the use of parameter-space searching techniques. We here use two-dimensional angle searching to estimate the DOA.

Consider $N$-component EmVS array $(2 \leq N \leq 6)$, then the matrix $\mathbf{A}_{\mathrm{e}}$ in (15) can be rewritten as $\mathbf{A}_{\mathrm{e}}=\left[\mathbf{A}_{\mathrm{e}, 1}^{T}\right.$, $\left.\ldots, \mathbf{A}_{e, N}^{T}\right]^{T} \in C^{N(L-1) \times K}$, and $\mathbf{A}_{e, n}$ can also be rewritten as

$$
\mathbf{A}_{\mathrm{e}, n}=\mathbf{Q}_{\mathrm{e}} \prod_{n}, \quad n=1, \ldots, N,
$$

where $\mathbf{Q}_{\mathrm{e}} \stackrel{\text { def }}{=}\left[\mathbf{q}_{\mathrm{e}}\left(\theta_{1}, \varphi_{1}\right), \ldots, \mathbf{q}_{\mathrm{e}}\left(\theta_{K}, \varphi_{K}\right)\right] \in C^{(L-1) \times K}, \prod_{n} \stackrel{\text { def }}{=}$ $\operatorname{diag}\left(c_{n, 1}, \ldots, c_{n, K}\right) \in C^{K \times K}$.
Defining $\mathbf{g}_{n} \stackrel{\text { def }}{=}\left[\mathbf{0}_{L-1,(L-1)(n-1)}, \mathbf{I}_{L-1}, \mathbf{0}_{L-1,(L-1)(N-n)}\right] \in$ $R^{(L-1) \times N(L-1)}$, we have $\mathbf{R}_{\mathrm{g}} \stackrel{\text { def }}{=} \sum_{n=1}^{N} \mathbf{g}_{n} \mathbf{R}_{\mathrm{e}}=\mathbf{Q}_{\mathrm{e}} \bar{\Pi} \boldsymbol{\Omega}$, where $\bar{\Pi} \stackrel{\text { def }}{=} \sum_{n=1}^{N} \Pi_{n}$. Partitioning $\mathbf{R}_{\mathrm{g}}$ into $\mathbf{R}_{\mathrm{g}}=\left[\mathbf{R}_{\mathrm{g} 1}^{T} \mathbf{R}_{\mathrm{g} 2}^{T}\right]^{T}$, where $\mathbf{R}_{\mathrm{g} 1}$ and $\mathbf{R}_{\mathrm{g} 2}$ consist of the first $K$ rows and the last $L-$ $1-K$ rows of $\mathbf{R}_{\mathrm{g}}$, we have the propagator matrix $\mathbf{P}=$ $\left(\mathbf{R}_{\mathrm{g} 1} \mathbf{R}_{\mathrm{g} 1}^{H}\right)^{-1} \mathbf{R}_{\mathrm{g} 1} \mathbf{R}_{\mathrm{g} 2}^{H}$. Then the source's DOA parameters can be estimated as

$$
\left\{\theta_{k}, \varphi_{k}\right\}=\underbrace{\arg \min }_{\{\theta, \varphi\}} \mathbf{q}_{\mathrm{e}}^{H}(\theta, \varphi) \Psi \Psi^{H} \mathbf{q}_{\mathrm{e}}(\theta, \varphi),
$$

where $\boldsymbol{\Psi} \stackrel{\text { def }}{=}\left[\mathbf{P}^{T},-\mathbf{I}_{L-1-K}\right]^{T}$.

\section{Simulations}

We conduct computer simulations to evaluate the performances of the proposed EVSA-PM. Comparison with the PSA based [19] PM (PSA-PM) and the SUMWE algorithm [26] is also made. For proposed EVSA-PM algorithm, the parameter estimates shown in Figures 1-5 are extracted from the EmVS steering vector, and those shown in Figure 6 are obtained by angle searching. The performance metrics used is the root mean square errors (RMSEs) of the sources' 2-D DOA and the polarization parameters estimates, where the RMSE of $k$ th source's 2-D DOA estimate is defined as

$$
\begin{aligned}
\operatorname{RMSE}_{k}= & \frac{1}{2}\left\{\sqrt{\frac{1}{E}\left(\sum_{e=1}^{E}\left(\hat{\theta}_{e, k}-\theta_{k}\right)^{2}\right)}\right. \\
& \left.+\sqrt{\frac{1}{E}\left(\sum_{e=1}^{E}\left(\hat{\varphi}_{e, k}-\varphi_{k}\right)^{2}\right)}\right\},
\end{aligned}
$$




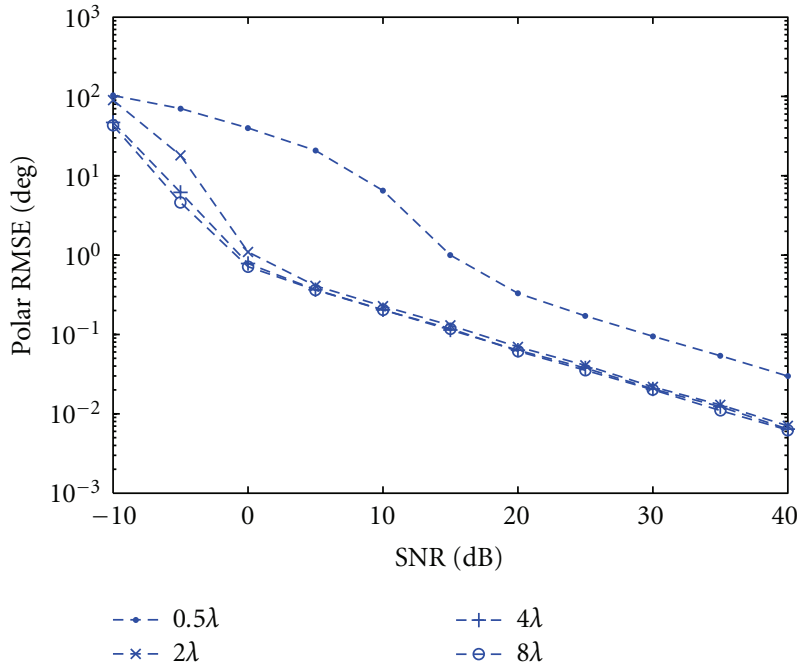

(a)

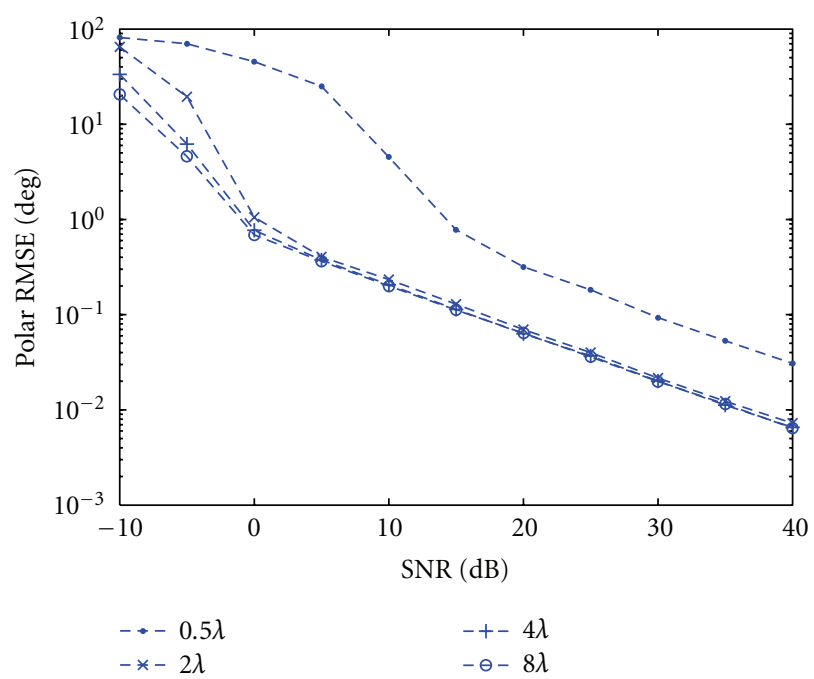

(b)

FIGURE 2: Polarization state estimates RMSE of the proposed EVSA-PM against SNRs. (a) Source 1, (b) source 2.

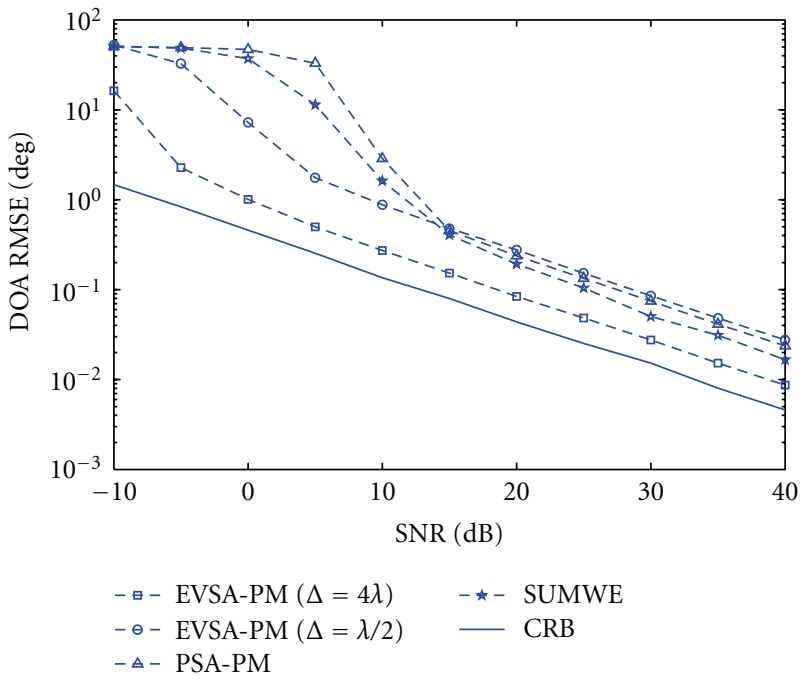

(a)

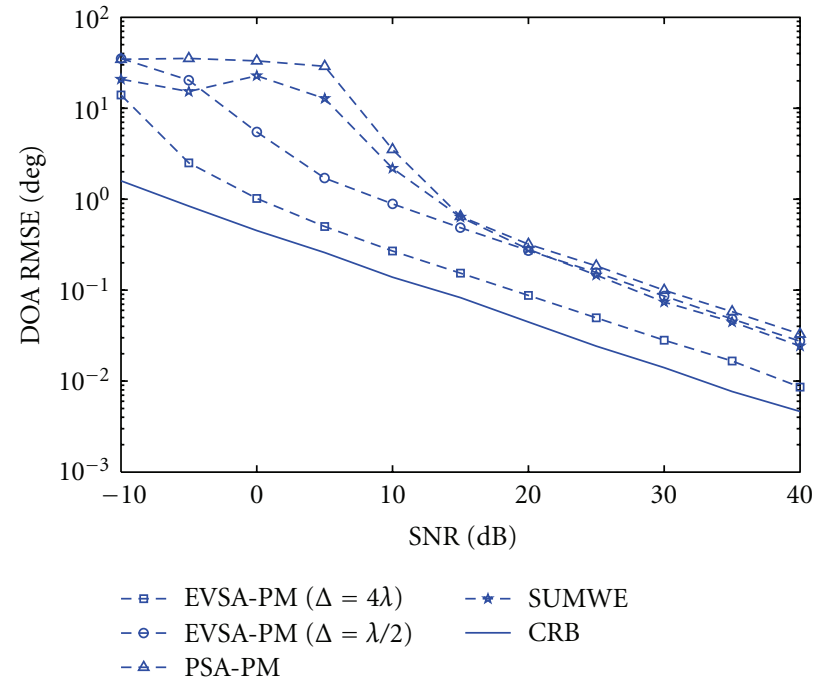

(b)

Figure 3: DOA estimate RMSEs of EVSA-PM, PSA-PM, and SUMWE against SNRs. (a) Source 1, (b) source 2.

and the RMSE of $k$ th source's polarization state estimate is defined as

$$
\begin{aligned}
\operatorname{RMSE}_{k}=\frac{1}{2}\left\{\sqrt{\frac{1}{E}\left(\sum_{e=1}^{E}\left(\hat{\gamma}_{e, k}-\gamma_{k}\right)^{2}\right)}\right. \\
\left.+\sqrt{\frac{1}{E}\left(\sum_{e=1}^{E}\left(\hat{\eta}_{e, k}-\eta_{k}\right)^{2}\right)}\right\},
\end{aligned}
$$

where $\hat{\theta}_{e, k}, \hat{\varphi}_{e, k}, \hat{\gamma}_{e, k}$, and $\hat{\eta}_{e, k}$ symbolize the eth Monte Carlo trial's estimates for the $k$ th source's directions and polarization states and $E$ is the total Monte Carlo trials. In the simulations, $E=500$.
Figures 1 and 2 plot the RMSEs of the sources' DOA and polarization estimates against signal-to-noise ratio (SNR) levels using the EVSA-PM. The SNR is defined as SNR = $(1 / K) \sum_{k=1}^{K}\left|s_{k}\right|^{2} / \sigma_{n}^{2}$, where $\sigma_{n}^{2}$ is the noise power lever. Two equal-power narrowband coherent signals impinge with parameters $\theta_{1}=75^{\circ}, \varphi_{1}=35^{\circ}, \gamma_{1}=45^{\circ}, \eta_{1}=-90^{\circ}, \theta_{2}=$ $80^{\circ}, \varphi_{2}=30^{\circ}, \gamma_{2}=45^{\circ}$, and $\eta_{2}=90^{\circ}$, and the multipath coefficient is set to $\beta_{2}=\exp \left(j * 50^{\circ}\right)$. The uniform linear array consists of 12 six-component EmVSs. The intervector sensor spacing is set as $\Delta=\sqrt{\Delta_{x}^{2}+\Delta_{y}^{2}}=0.5 \lambda, 2 \lambda, 4 \lambda$, and $8 \lambda$, respectively. The snapshot number is 300 . It is seen from that both DOA and polarization estimation errors decreases as the SNR increases. Also, the increase of intervector sensor spacing, which results in the array aperture extension, 


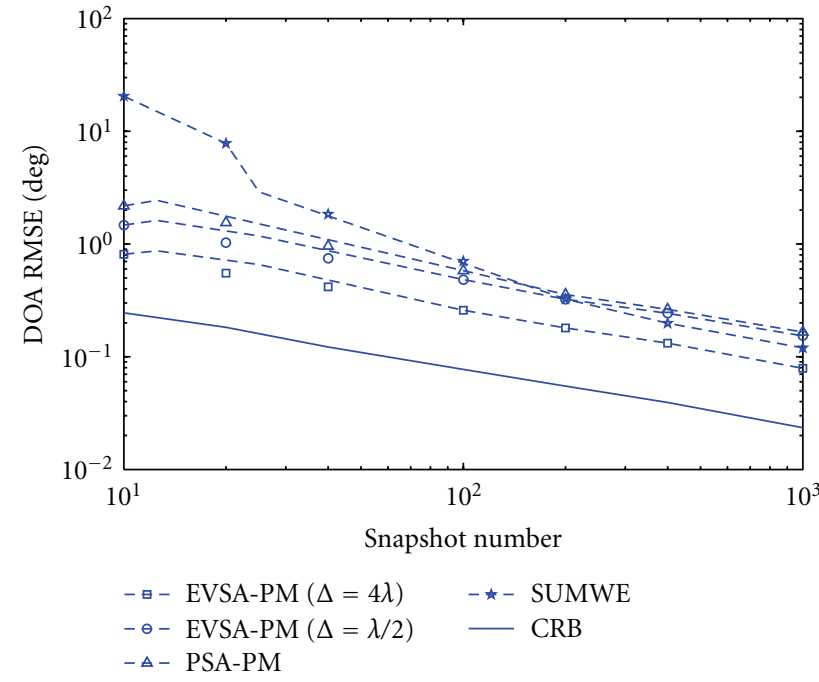

(a)

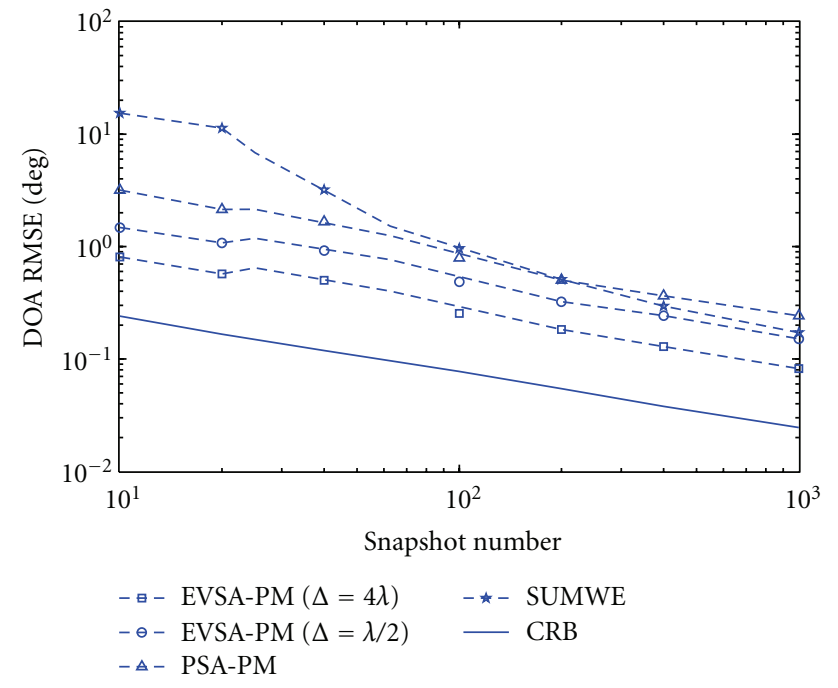

(b)

FIGURE 4: DOA estimate RMSEs of EVSA-PM, PSA-PM and SUMWE against the number of snapshots. (a) Source 1, (b) source 2.

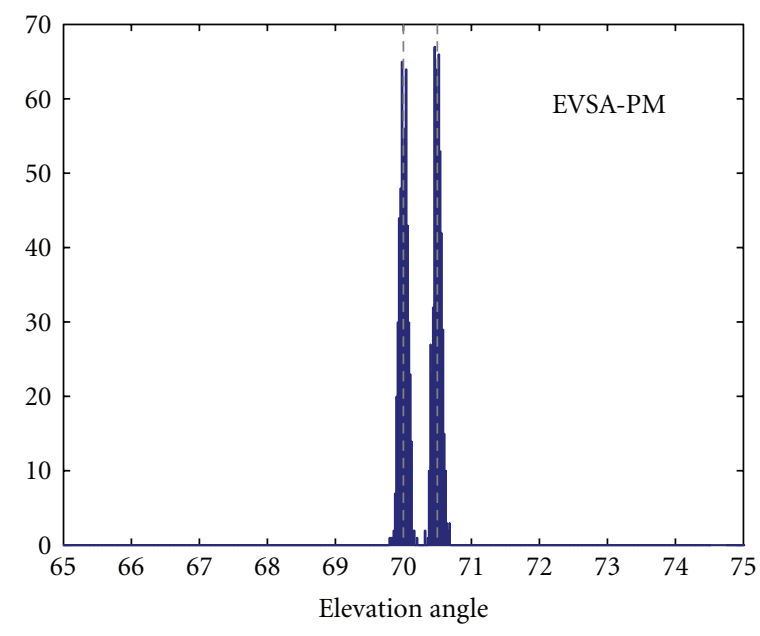

(a)

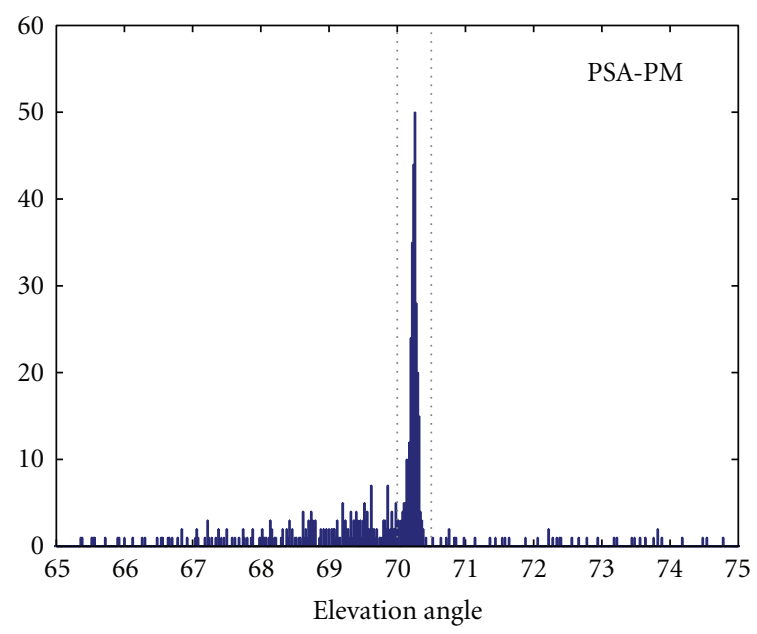

(b)

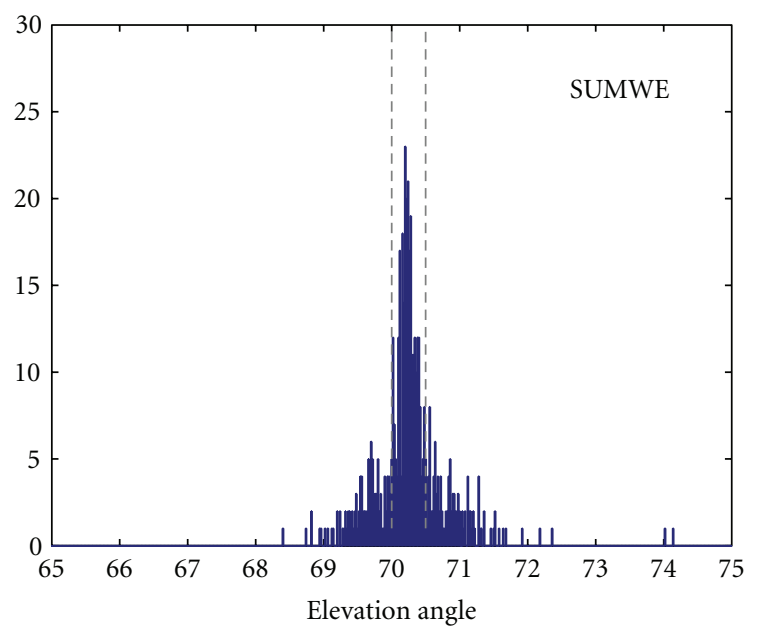

(c)

FIGURE 5: The histogram of the estimated elevation using the three methods. (a) EVSA-PM; (b) PSA-PM; (c) SUMWE. 
contributes to the estimation accuracy enhancement. Since the estimation of DOA and polarization is extracted from the EmVS steering vector, which contains no time-delay phase factor, we can obtain more accurate but unambiguous estimates of coherent source using an aperture extension array without a corresponding increase in hardware and software costs [12].

Figures 3 and 4 make the comparison between the proposed algorithm with PSA-PM and SUMWE under different SNRs and number of snapshots. The impinging signal parameters are same as in Figures 1 and 2. We use 300 snapshots in Figure 3 and set SNR $=20 \mathrm{~dB}$ in Figure 4. For the proposed algorithm, a uniform linear array with 8 dipole-triads, separated by $\Delta=\lambda / 2$ and $4 \lambda$ is considered. For the PSA-PM, we use an L-shape geometry, with 8 dipoletriads uniformly placed along $x$-axis for estimating $u_{k}$ and 8 dipole-triads uniformly placed along $y$-axis for estimating $v_{k}$. For the SUMWE, we use an L-shape geometry, with 12 unpolarized scalar sensors uniformly placed along $x$ axis for estimating $u_{k}$ and 12 unpolarized scalar sensors uniformly placed along $y$-axis for estimating $v_{k}$. Hence, the hardware costs of the SUMWE and the presented algorithm are comparable. The intersensor displacement for the PSA$\mathrm{PM}$ and SUMWE is a half-wavelength, since these two algorithms would suffer angle ambiguities when two sensors are spaced over a half-wavelength. The curves in these two figures unanimously demonstrate that the proposed EVSAPM with $\Delta=4 \lambda$ can offer performance superior to those of the PSA-PM and SUMWE.

From the computational complexity analysis, the major computational costs involved in the three algorithms are the calculation of the corresponding propagator and correlation matrix, and the numbers of multiplications required by the EVSA-PM, the PSA-PM, and SUMWE are in the order of $O\left(3 M_{1} K F+18\left(M_{1}-1\right) F\right) \approx 174 F, O\left(2 M_{1} K F+6 M_{1}^{2} F\right) \approx$ $416 F$, and $O\left(2 M_{2} K F+4\left(M_{2}-1\right) F\right) \approx 92 F$, respectively, where $M_{1}=8, M_{2}=12$, and $F$ denotes the number of snapshots. Therefore, the proposed EVSA-PM also is more computationally efficient than the PSA-PM.

The proposed EVSA-PM can fully exploit polarization diversity to resolve closely spaced sources with distinct polarizations. To verify this performance, we assume two incident coherent sources with parameters $\theta_{1}=70^{\circ}, \theta_{2}=$ $70.5^{\circ}, \varphi_{1}=90^{\circ}, \varphi_{2}=90^{\circ}, \gamma_{1}=45^{\circ}, \gamma_{2}=45^{\circ}, \eta_{1}=-90^{\circ}$, and $\eta_{2}=90^{\circ}$. Others simulation conditions are the same as that in Figure 4, except that the SNR is set at $35 \mathrm{~dB}$. Figure 5 shows the histogram of the estimated elevation using the three methods based on 500 independent trials. From the figure, we can observe that the proposed EVSA-PM can resolve the closely spaced sources. However, the other two methods fail.

Figure 6 plots the spatial spectrum to present comparison of the maximum numbers of coherent signals, which can be, respectively, resolved by the proposed algorithm, the SUMWE, the PSA-PM, and the PSA-FB-PM which combines the PSA with the FB averaging technique [27]. We consider a uniform linear array comprised of 20 unpolarized scalar sensors for the SUMWE and 20 quadrature polarized vector

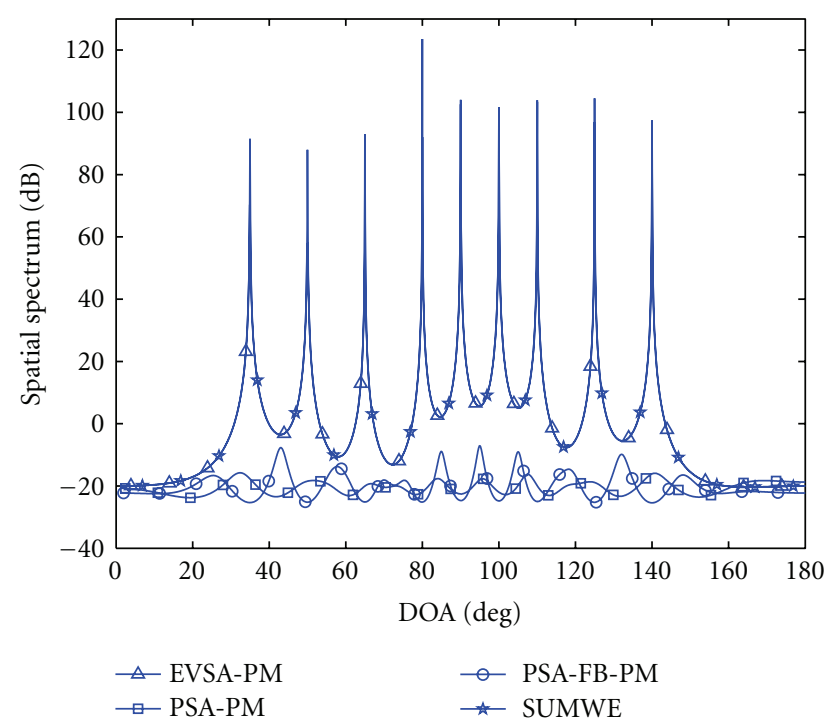

Figure 6: Spatial spectrum of EVSA-PM, PSA-PM, PSA-FB-PM, and SUMWE for nine coherent sources.

sensors [19] (i.e., $N=4, M=20$ ) for all the other three algorithms and estimate the sources' direction by angle searching. The intervector sensor spacing of array is a halfwavelength. Like [19], we assume zero elevation incident angle $\left(\theta_{k}=90^{\circ}\right)$ and randomly chosen polarizations for all sources, and set $\mathrm{SNR}=15 \mathrm{~dB}$.

Nine equal power, coherent sources with the azimuth incident angles $35^{\circ}, 50^{\circ}, 65^{\circ}, 80^{\circ}, 90^{\circ}, 100^{\circ}, 110^{\circ}, 125^{\circ}$, and $140^{\circ}$ are considered, and the corresponding multipath coefficients $\beta_{k}=\exp \left(j * 10^{\circ}(k-1)\right), k=1, \ldots, 9$. This figure shows that the proposed EVSA-PM and the SUMWE successfully resolve the nine coherent signals, while the PSAPM, and the PSA-FB-PM fail to do so. This is due to the factor that the PSA-PM and the PSA-FB-PM, respectively, only can resolve $\min (N, M-1)=4$ and $\min (2 N, M-1)=8$ coherent sources at most, while the proposed EVSA-PM can resolve $L-2$ coherent sources $(L=M-K+1)$, and the maximum number of coherent signals resolved using the SUMWE is equal to that using the EVSA-PM.

\section{Conclusions}

This paper employs a linear electromagnetic vector-sensor array to propose a novel pre-processing algorithm for decorrelating the coherent signals by electromagnetic vectorsensor subarray averaging, and combine it with the propagator method to estimate the DOA and polarization of coherent sources without eigen-decomposition into signal/noise subspaces. Compared with the existing estimate algorithms, the proposed algorithm makes use of more available electromagnetic information, hence, has an improved estimation performance. It does not necessarily require the intervector sensor spacing of a half-wavelength, enable decorrelation of more coherent signals, and joint estimation of DOA and polarization of coherent sources. 


\section{Appendix}

From (12), we can obtain

$$
\left[\mathbf{R}_{1}, \ldots, \mathbf{R}_{6}\right] \stackrel{\text { def }}{=} \widetilde{A} F G,
$$

where $\mathrm{F} \stackrel{\text { def }}{=} \operatorname{diag}\left(\mathrm{r}_{\mathrm{s}_{1}} \beta_{1}, \ldots, \mathrm{r}_{\mathrm{s}_{1}} \beta_{\mathrm{K}_{1}}, \mathrm{r}_{\mathrm{S}_{\mathrm{K}_{1}+1}} \mathrm{q}_{\mathrm{M}}\left(\theta_{\mathrm{K}_{1}+1}, \varphi_{\mathrm{K}_{1}+1}\right), \ldots\right.$, $\left.\mathrm{r}_{\mathrm{s}_{\mathrm{K}}} \mathrm{q}_{\mathrm{M}}\left(\theta_{\mathrm{K}}, \varphi_{\mathrm{K}}\right)\right)$

$$
\mathrm{G} \stackrel{\text { def }}{=}\left[\begin{array}{cccc}
\tilde{\rho}_{\mathrm{M}, 1} \mathrm{~h}_{1}^{\mathrm{T}} & \tilde{\rho}_{\mathrm{M}, 2} \mathrm{~h}_{1}^{\mathrm{T}} & \ldots & \tilde{\rho}_{\mathrm{M}, 6} \mathrm{~h}_{1}^{\mathrm{T}} \\
\vdots & \vdots & \ddots & \vdots \\
\tilde{\rho}_{\mathrm{M}, 1} \mathrm{~h}_{\mathrm{K}_{1}}^{\mathrm{T}} & \tilde{\rho}_{\mathrm{M}, 2} \mathrm{~h}_{\mathrm{K}_{1}}^{\mathrm{T}} & \ldots & \tilde{\rho}_{\mathrm{M}, 6} \mathrm{~h}_{\mathrm{K}_{1}}^{\mathrm{T}} \\
\mathrm{c}_{1, \mathrm{~K}_{1}+1} \mathrm{~h}_{\mathrm{K}_{1}+1}^{\mathrm{T}} & \mathrm{c}_{2, \mathrm{~K}_{1}+1} \mathrm{~h}_{\mathrm{K}_{1}+1}^{\mathrm{T}} & \ldots & \mathrm{c}_{6, \mathrm{~K}_{1}+1} \mathrm{~h}_{\mathrm{K}_{1}+1}^{\mathrm{T}} \\
\vdots & \vdots & \ddots & \vdots \\
\mathrm{c}_{1, \mathrm{~K}} \mathrm{~h}_{\mathrm{K}}^{\mathrm{T}} & \mathrm{c}_{2, \mathrm{~K}} \mathrm{~h}_{\mathrm{K}}^{\mathrm{T}} & \ldots & \mathrm{c}_{6, \mathrm{~K}} \mathrm{~h}_{\mathrm{K}}^{\mathrm{T}}
\end{array}\right],
$$

The matrix $\tilde{\mathbf{A}}$ is of full column rank due to the distinct polarizations (although there are two sources from the same direction). The diagonal matrix $\mathbf{F}$ has full rank. If the two sources have the same incident directions but with the distinct polarizations, and are uncorrelated with each other (i.e., the two sources are not all included in the set consisting of the first $K_{1}$ coherent sources), the $K \times 6 K$ matrix $\mathbf{G}$ is of full row rank. Therefore, in this scenario, the matrix $\left[\mathbf{R}_{1}, \ldots, \mathbf{R}_{6}\right]$ is of rank $K$. Similarly, the matrix $\left[\widetilde{\mathbf{R}}_{1}, \ldots, \widetilde{\mathbf{R}}_{6}\right]$ also is of rank $K$. Thus, the matrix $\mathbf{R}$ defined in (11) still has full rank.

\section{References}

[1] A. Nehorai and E. Paldi, "Vector-sensor array processing for electromagnetic source localization," IEEE Transactions on Signal Processing, vol. 42, no. 2, pp. 376-398, 1994.

[2] J. Li, "Direction and polarization estimation using arrays with small loops and short dipoles," IEEE Transactions on Antennas and Propagation, vol. 41, no. 3, pp. 379-386, 1993.

[3] K. T. Wong, "Direction finding/polarization estimationdipole and/or loop triad(s)," IEEE Transactions on Aerospace and Electronic Systems, vol. 37, no. 2, pp. 679-684, 2001.

[4] B. Hochwald and A. Nehorai, "Polarimetric modeling and parameter estimation with applications to remote sensing," IEEE Transactions on Signal Processing, vol. 43, no. 8, pp. 19231935, 1995.

[5] X. Gong, Z. Liu, Y. Xu, and M. Ishtiaq Ahmad, "Directionof-arrival estimation via twofold mode-projection," Signal Processing, vol. 89, no. 5, pp. 831-842, 2009.

[6] J. Tabrikian, R. Shavit, and D. Rahamim, "An efficient vector sensor configuration for source localization," IEEE Signal Processing Letters, vol. 11, no. 8, pp. 690-693, 2004.

[7] S. Miron, N. Le Bihan, and J. I. Mars, "Quaternion-MUSIC for vector-sensor array processing," IEEE Transactions on Signal Processing, vol. 54, no. 4, pp. 1218-1229, 2006.

[8] C. C. Ko, J. Zhang, and A. Nehorai, "Separation and tracking of multiple broadband sources with one electromagnetic vector sensor," IEEE Transactions on Aerospace and Electronic Systems, vol. 38, no. 3, pp. 1109-1116, 2002.
[9] C. Paulus and J. I. Mars, "Vector-sensor array processing for polarization parameters and DOA estimation," EURASIP Journal on Advances in Signal Processing, vol. 2010, Article ID 850265, 3 pages, 2010.

[10] Y. Xu, Z. Liu, K. T. Wong, and J. Cao, "Virtual-manifold ambiguity in HOS-based direction-finding with electromagnetic vector-sensors," IEEE Transactions on Aerospace and Electronic Systems, vol. 44, no. 4, pp. 1291-1308, 2008.

[11] K. T. Wong and M. D. Zoltowski, "Closed-form direction finding and polarization estimation with arbitrarily spaced electromagnetic vector-sensors at unknown locations," IEEE Transactions on Antennas and Propagation, vol. 48, no. 5, pp. 671-681, 2000.

[12] M. D. Zoltowski and K. T. Wong, "ESPRIT-based 2-D direction finding with a sparse uniform array of electromagnetic vector sensors," IEEE Transactions on Signal Processing, vol. 48, no. 8, pp. 2195-2204, 2000.

[13] K. T. Wong, "Blind beamforming geolocation for widebandFFHs with unknown hop-sequences," IEEE Transactions on Aerospace and Electronic Systems, vol. 37, no. 1, pp. 65-76, 2001.

[14] H. Jiacai, S. Yaowu, and T. Jianwu, "Joint estimation of DOA, frequency, and polarization based on cumulants and UCA," Journal of Systems Engineering and Electronics, vol. 18, no. 4, pp. 704-709, 2007.

[15] K. C. Ho, K. C. Tan, and A. Nehorai, "Estimating directions of arrival of completely and incompletely polarized signals with electromagnetic vector sensors," IEEE Transactions on Signal Processing, vol. 47, no. 10, pp. 2845-2852, 1999.

[16] K. T. Wong and M. D. Zoltowski, "Uni-vector-sensor ESPRIT for multisource azimuth, elevation, and polarization estimation," IEEE Transactions on Antennas and Propagation, vol. 45, no. 10, pp. 1467-1474, 1997.

[17] K. T. Wong and M. D. Zoltowski, "Self-initiating MUSICbased direction finding and polarization estimation in spatiopolarizational beamspace," IEEE Transactions on Antennas and Propagation, vol. 48, no. 8, pp. 1235-1245, 2000.

[18] M. D. Zoltowski and K. T. Wong, "Closed-form eigenstructure-based direction finding using arbitrary but identical subarrays on a sparse uniform Cartesian array grid," IEEE Transactions on Signal Processing, vol. 48, no. 8, pp. 2205-2210, 2000.

[19] D. Rahamim, J. Tabrikian, and R. Shavit, "Source localization using vector sensor array in a multipath environment," IEEE Transactions on Signal Processing, vol. 52, no. 11, pp. 30963103, 2004.

[20] Y. Wu, H. C. So, C. Hou, and J. Li, "Passive localization of near-field sources with a polarization sensitive array," IEEE Transactions on Antennas and Propagation, vol. 55, no. 8, pp. 2402-2408, 2007.

[21] M. Kanda and D. A. Hill, "A three-loop method for determining the radiation characteristics of an electrically small source," IEEE Transactions on Electromagnetic Compatibility, vol. 34, no. 1, pp. 1-3, 1992.

[22] R. O. Schmidt, "Multiple emitter location and signal parameter estimation," IEEE Transactions on Antennas and Propagation, vol. 34, no. 3, pp. 276-280, 1986.

[23] R. Roy and T. Kailath, "ESPRIT_estimation of signal parameters via rotational invariance techniques," IEEE Transactions on Acoustics, Speech, and Signal Processing, vol. 37, no. 7, pp. 984995, 1989. 
[24] N. Tayem and H. M. Kwon, "L-shape 2-dimensional arrival angle estimation with propagator method," IEEE Transactions on Antennas and Propagation, vol. 53, no. 5, pp. 1622-1630, 2005.

[25] C. Gu, J. He, X. Zhu, and Z. Liu, "Efficient 2D DOA estimation of coherent signals in spatially correlated noise using electromagnetic vector sensors," Multidimensional Systems and Signal Processing, vol. 21, no. 3, pp. 239-254, 2010.

[26] J. Xin and A. Sano, "Computationally efficient subspacebased method for direction-of-arrival estimation without eigendecomposition," IEEE Transactions on Signal Processing, vol. 52, no. 4, pp. 876-893, 2004.

[27] S. U. Pillai and B. H. Kwon, "Forward/backward spatial smoothing techniques for coherent signal identification," IEEE Transactions on Acoustics, Speech, and Signal Processing, vol. 37, no. 1 , pp. 8-15, 1989. 